\title{
PERFORMANCE BENEFITS WITH SCENE-LINKED HUD SYMBOLOGY: AN ATTENTIONAL PHENOMENON?
}

\author{
Jonathan L. Levy \\ University of California, San Diego \\ La Jolla, CA
}

\author{
David C. Foyle \\ NASA Ames Research Center \\ Moffett Field, CA
}

\author{
Robert S. McCann \\ San Jose State University \\ Moffett Field, CA
}

Previous research has shown that in a simulated flight task, navigating a path defined by ground markers while maintaining a target altitude is more accurate when an altitude indicator appears in a virtual "scenelinked" format (projected symbology moving as if it were part of the out-the-window environment) compared to the fixed-location, superimposed format found on present-day HUDs (Foyle, McCann \& Shelden, 1995). One explanation of the scene-linked performance advantage is that attention can be divided between scene-linked symbology and the outside world more efficiently than between standard (fixedposition) HUD symbology and the outside world. The present study tested two alternative explanations by manipulating the location of the scene-linked HUD symbology relative to the ground path markers. Scenelinked symbology yielded better ground path-following performance than standard fixed-location superimposed symbology regardless of whether the scene-linked symbology appeared directly along the ground path or at various distances off the path. The results support the explanation that the performance benefits found with scene-linked symbology are attentional.

\section{INTRODUCTION}

Piloting an aircraft consists of many subtasks. For example, at any given moment the pilot might be trying to navigate a path, maintain a particular altitude, visually scan the environment, and monitor radio transmissions. In order to accomplish these tasks, the pilot must process many stimuli (e.g., the landscape, gauges, auditory messages). From a psychological standpoint, each processing demand places an additional load on human attention. Because the capacity of human attention is limited, an important goal of human factors researchers is to design displays that minimize the information processing demands on the operator.

One method widely thought to reduce the effort needed to acquire flight-relevant information from cockpit instruments is to project information normally found on head-down instrument panels onto a Head-Up Display (HUD). A HUD is a collimated, transparent display medium upon which instrument symbology is shown directly superimposed on the out-the-window scene. HUDs were designed to eliminate the need to refocus the eyes with their collimated optics, and the need for large eye-scan movements between the out-thewindow view and the instrument panel, since the out-thewindow scene and the HUD symbology can be placed near one another. In addition, the direct superimposition of HUD symbology on the outside world makes it physically possible for the pilot to process both sources of information simultaneously.

Although flight-related performance benefits using HUDs are well documented (e.g., Boucek, Pfaff \& Smith, 1983; MartinEmerson \& Wickens, 1997), recent results call into question whether superimposed HUD symbology facilitates the joint processing of instrument symbology and the out-the-window scene. In a simulated landing experiment, Wickens and Long
(1995) found that pilots took longer to notice a potential runway incursion and initiate a go-around when using a HUD compared to a head-down panel display (also see Fischer, Haines \& Price, 1980). These results suggest that, despite the design goal of HUDs, pilots did not maintain simultaneous awareness of HUD symbology (what Wickens calls the "near domain") and the out-the-window scene (the "far domain"). Furthermore, in a terrain flight simulation, Foyle, McCann and Shelden (1995) had subjects perform two continuous, simultaneous tasks in wind turbulence conditions: Follow a path defined by a winding series of ground-markers and maintain a target altitude of 100 feet. In one condition, the no-gauge baseline condition, current altitude had to be estimated using only the natural, perspective cues in the outthe-window scene (e.g., ground objects varied in size as a function of altitude). In another condition, these natural, perspective cues were supplemented by a HUD-like readout (digital or analog) of current altitude superimposed at a fixed location on the screen. Not surprisingly, subjects were better able to maintain the target altitude when an altitude gauge was present than when it was absent. This improvement in altitude maintenance, however, was accompanied by an associated reduction in path-following accuracy: Pathfollowing performance was worse when a fixed-screen location superimposed altitude gauge was present than when it was absent. Foyle, McCann and Shelden (1995) referred to this performance pattern as the altitude/path performance tradeoff.

One explanation of both the Wickens and Long (1995) findings and the Foyle, McCann and Shelden (1995) tradeoff is that they reflect limitations of human visual/spatial attention. There is considerable evidence to suggest that, while it is possible to divide attention among stimuli that group together on the basis of salient perceptual characteristics (e.g., common 
motion, color, shape), it is difficult to divide auention across stimuli belonging to separate perceptual groups (Kahneman \& Henik, 1981). There are a number of salient visual cues that would bias the visual system to parse the HUD as one perceptual group, and the world as another. For example, most HUD symbology is stationary (i.e., appearing at a fixed location on the display) whereas elements in the far domain appear to be in continual motion with respect to the observer in a moving aircraft. Assuming this parsing/grouping of the near and far domains occurs, it would discourage or prevent attention from being efficiently divided between HUD symbology and the out-the-window scene. Thus, when subjects are attending to fixed-location superimposed HUD symbology, unexpected events in the far domain are difficult to detect (Wickens \& Long, 1995) and deviations from the ground path take longer to notice and be corrected (Foyle, McCann \& Shelden, 1995).

Guided by these considerations, Foyle, McCann and Shelden (1995) developed a potential design solution to the parsing problem in the form of "scene-linked symbology" -symbology was projected at a specific location in the scene, so as one moves through the world, scene-linked symbology undergoes the same optical transformations as real-world objects do, giving the symbology the appearance of being a real-world object itself. According to attentional theory, scenelinked symbology should group with the far domain, thereby enabling the efficient division of attention between HUD symbology and the far domain.

If the altitude/path performance tradeoff found with the fixedlocation superimposed HUD altitude gauge is an attentional problem, presenting the altitude gauge in a scene-linked form should eliminate the tradeoff. To test this hypothesis, Foyle, McCann and Shelden (1995) compared performance in their simulation when the altitude gauge was superimposed (and thus stationary, in a fixed location on the display) to a condition in which altitude gauges were scene-linked and located along the path, directly between the ground markers. Compared to the no-gauge baseline condition, performance on the altitude task showed equivalent improvement with the traditional superimposed HUD and the scene-linked altitude gauges. As in earlier studies (for a review, see McCann \& Foyle, 1995), the fixed-location superimposed symbology led to an altitude/path performance tradeoff: The improvement in altitude maintenance was accompanied by worse pathfollowing performance. In sharp contrast, not only was the performance tradeoff not observed with the scene-linked gauges, but path-following error was actually significantly smaller than in either the superimposed symbology or the noHUD baseline conditions.

According to Foyle, McCann and Shelden (1995), the improvement in path-following performance with the scenelinked gauges was the result of being able to divide atuention efficiently between the scene-linked gauges and the outside world, something that was not possible with the superimposed symbology gauge. However, there are other explanations as well. In the scene-linked condition, 18 altitude gauges appeared on the path among the 37 markers; thus, the path was defined by a total of 55 items instead of only 37 as in the superimposed-and no-gauge baseline conditions. The reduction in path-following error may have occurred simply because the path was better defined in the scene-linked condition (termed the better-defined path explanation). Furthermore, because the ground path-markers were arranged to define a winding path, they were seen at various locations on the monitor as one flew over them. As a result, the pathmarkers varied in physical screen distance from the superimposed, screen-centered altitude gauge. The scene-linked gauges always appeared directly on the path, so the screendistance between the scene-linked gauges and the path-markers was typically smaller than between the fixed-location superimposed HUD symbology gauge and the path markers. Thus, better path performance in the scene-linked condition could have resulted because the altitude gauge information was in closer proximity (on the monitor) to path information in the scene-linked condition than in the superimposed symbology condition (termed the visual proximity explanation).

The goal of the present experiment was to test the validity of these two non-attentional explanations of the scene-linked symbology performance benefit (i.e., not only the elimination of the altitude/path performance tradeoff, but better than baseline path-following performance with scene-linked symbology). Each subject flew a simulated aircraft in a series of short flights. On each flight, subjects were required to fly directly over a winding path defined by a series of equallyspaced ground markers and simultaneously maintain a target altitude of $100 \mathrm{ft}$. The two dependent measures were root mean square error (RMSE) deviation from the path (lateral offset) and RMSE deviation from $100 \mathrm{ft}$ (vertical offset). The single independent variable, the format of the altitude gauge, had five levels: No-gauge baseline condition; Superimposed symbology (the gauge at a fixed-display location); and, Three configurations of scene-linked symbology. The 18 scenelinked symbology gauges appeared directly along the path (as in Foyle, McCann \& Shelden, 1995), at a fixed lateral distance $(100 \mathrm{ft})$ to either side of the path, or at a random variable lateral distance $(50,70,90,110,130$, or $150 \mathrm{ft})$ off the path (see Figure 1).

The different scene-linked conditions allow us to discriminate between the attentional (i.e., attentional grouping) and the two non-attentional (better-defined path and visual proximity) explanations of the path performance benefits reported by Foyle, McCann and Shelden (1995). Following that study, all three explanations predict better path performance with the on-path scene-linked symbology altitude gauges compared to the superimposed symbology gauge and no-gauge baseline conditions. However, the three explanations make divergent predictions about performance with the fixedand variable-distance off-path scene-linked symbology gauges. Because the gauges were not placed directly along the path, it cannot be argued that they defined the path better, as when placed directly along the path (as in the scene-linked on-path 
symbology condition). Likewise, the distance (i.e., visual angle) between the path markers and the off-path gauges was roughly comparable to the distance between the path markers and the superimposed symbology gauge. Therefore, both the better-defined path and the visual proximity explanations predict equivalent path performance between these two off-path scene-linked symbology conditions and the superimposed symbology condition. In addition, the better-defined path and the yisual proximity explanations predict that performance in the on-path scene-linked symbology condition should be better than in the other conditions.

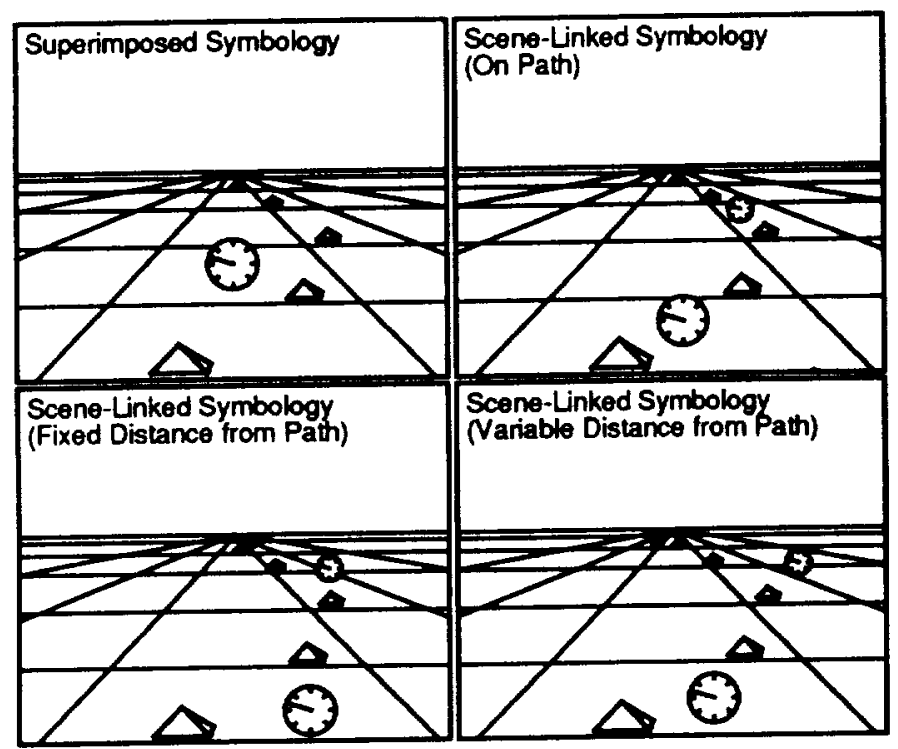

Figure 1. Schematic drawings (not to scale) of four altitude gauge symbology formats (the No-Gauge baseline condition is not shown).

By contrast, the attentional grouping explanation posits that the inherent qualities (e.g., lack of differential motion) of scene-linked symbology altitude gauges is the important factor in producing better-than-baseline path performance, not the physical relationship of the symbology gauges to the path per se. As a result, attention should be divided efficiently between path-markers and altitude gauges as long as they are scenelinked, regardless of the gauges' location. Empirically, pathfollowing performance should be equivalent in all three scenelinked conditions, and better than in the superimposed or nogauge baseline conditions.

\section{METHOD}

The flight simulation was controlled by a Silicon Graphics Indigo2 Impact computer. Images were displayed on a highresolution 19-inch color monitor placed $65 \mathrm{~cm}$ in front of a chair in a sound-attenuated, dimly-illuminated booth. The seated subjects controlled the flight with a spring-centered joystick, mounted on the chair's right armrest. Lateral joystick deflections controlled lateral, path-following movement while forward-backward deflections (down and up, respectively) controlled altitude.
The flight simulation, a simple kinematic model, was pitchstabilized (i.e., it did not pitch up or down when climbing or descending), thereby ensuring that the path information in the virtual environment would be visually available at all times. Roll was accurately depicted. The upper portion of the monitor was blue (representing the sky) and the lower portion was green (representing the ground). A white grid was superimposed on the ground (see Figure 1). The HUD gauge was a round, yellow circle with a black, clock-like am that rotated to indicate altitude (after Weinstein, Ercoline, Evans \& Bitton, 1992). In the superimposed symbology HUD condition, the gauge, located in the center of the monitor, measured $2.0 \mathrm{~cm}$ wide $\times 2.0$ high $\mathrm{cm}$. Items located on the ground (path-markers and scene-linked gauges) varied appropriately in size as a function of perspective. The brown pyramid-shaped path markers were $24 \mathrm{ft}$ square by $6 \mathrm{ft}$ high, and with the aircraft at an altitude of $100 \mathrm{ft}$, ranged in screen size from $0.5 \times 0.2 \mathrm{~cm}$ to $4.0 \times 1.5 \mathrm{~cm}$, depending on the forward distance between the aircraft and the symbology. Similarly, the diameter of the circular scene-linked gauges was $15 \mathrm{ft}$ and ranged from 0.5 to $3.5 \mathrm{~cm}$ in screen size.

All fourteen subjects were right-handed male university students who reported normal or corrected-to-normal vision. Each was paid for participating in the experiment which lasted, including rest periods, about 2.5 hrs. The experiment was composed of 18 blocks of trials. In each block, there were five trials (one per HUD condition), presented in random order. A session thus contained 90 trials. For the first $16 \mathrm{sec}$ of the trial, the aircraft flew in a straight-line trajectory at $100 \mathrm{ft}$-the target altitude -- towards the first path marker demarcating the beginning of one of eight randomly selected paths. This 10-sec period allowed the subject to calibrate to the goal altitude. For the remaining $40 \mathrm{sec}$ of flight, simulated air turbulence caused both lateral and vertical displacement of the aircraft's position, thereby requiring constant monitoring and positioning of the craft. Forward speed, however, was set to a constant 160 kts.

The experimenter emphasized the equal importance of the two tasks - navigate the craft over the path and simultaneousiy maintain the altitude at $100 \mathrm{ft}$-- and then demonstrated the task by flying three trials. The subject's performance was viewed via a repeating monitor located outside of the experiment booth. For the first 30 trials, the experimenter provided verbal feedback via a two-way (handsfree) speaker system and answered questions at the conclusion of each trial. Throughout the experiment, after each trial, path and altitude RMSE scores were visually presented to the subject.

\section{RESULTS}

The first eight blocks were considered practice, and scores from these blocks were not analyzed. Excluding these blocks, each subject's path-error scores were grouped across HUD condition and examined for outliers. One subject produced a path score (330 ft RMSE) -- over six standard deviations greater than his mean path score (with this score, $M=74.19$, 
S.D. $=40.29)$. This trial was considered an outlier and excluded from analysis; no other scores were omitted. For each subject, altitude and path scores were each averaged across blocks for each HUD condition; these data can be seen in Figures 2 and 3, respectively. Separate analyses were conducted for altitude and path performance.

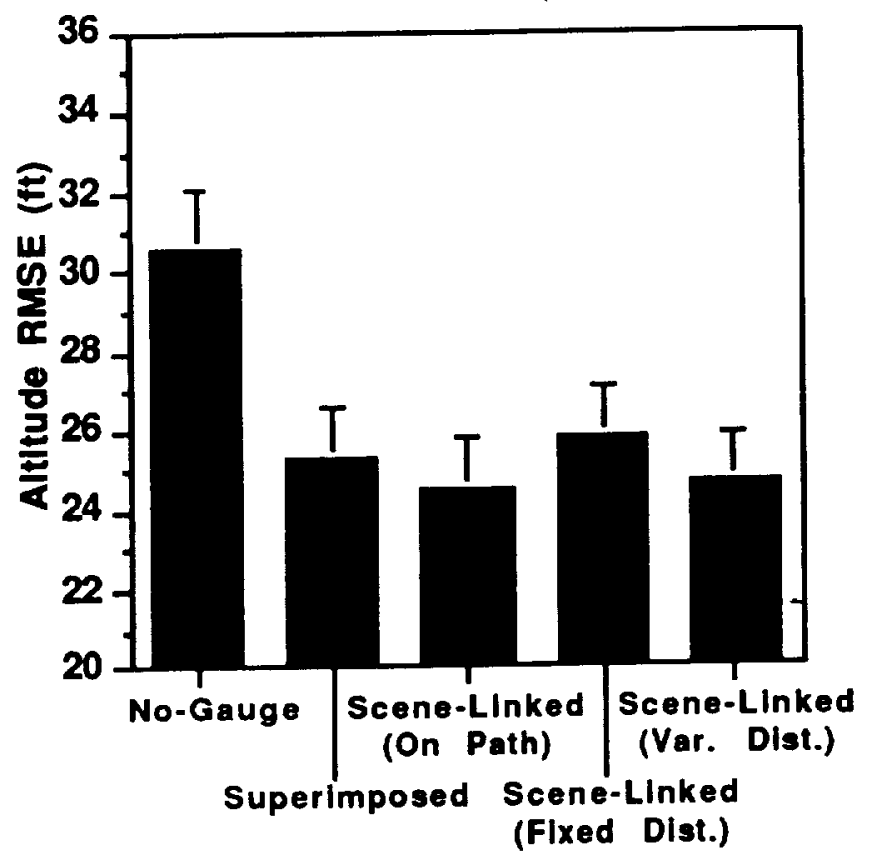

Figure 2. Altitude maintenance error (RMSE $\mathrm{ft}$ ) for the five altitude gauge format conditions.

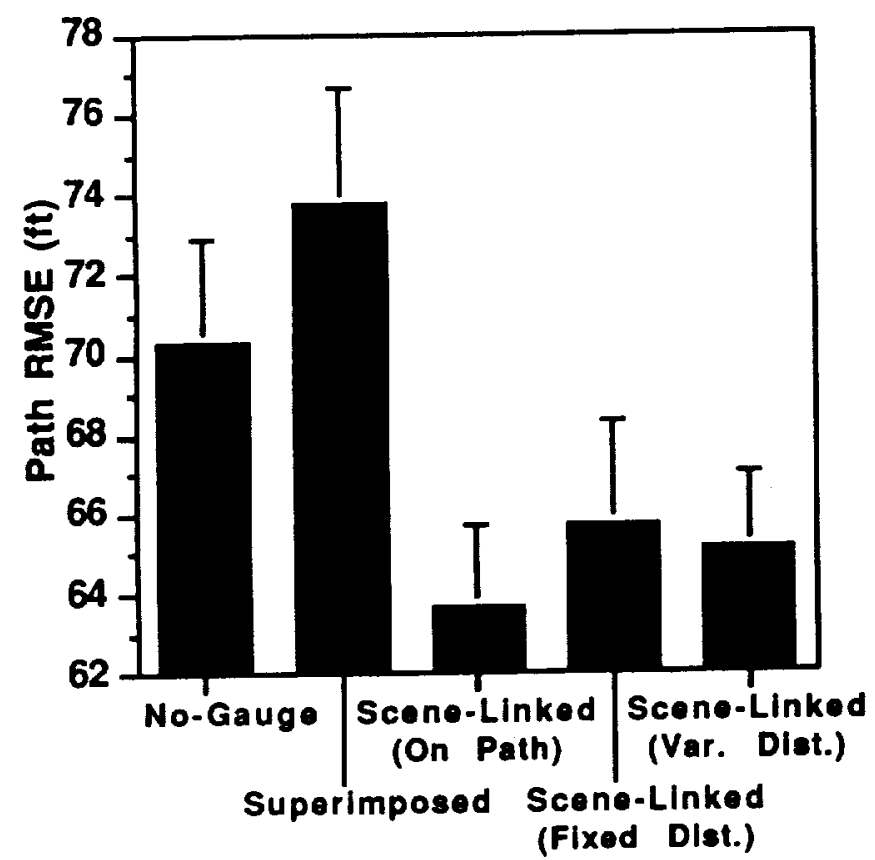

Figure 3. Path maintenance error (RMSE $\mathrm{ft}$ ) for the five altitude gauge format conditions.

Altitude performance

An analysis of variance (ANOVA) revealed a main effect of altitude gauge format on altitude scores, $F(4,52)=18.878$, $p<.001$. Additionally, Newman-Keuls planned comparisons $(\alpha=.05)$ showed there was no significant difference among the three scene-linked symbology conditions, $W_{\text {obeinod }} 1.28<$ $\mathrm{W}_{\text {crivicel }} 1.99, \mathrm{q}_{\cos }(3,52)$, and no significant difference among the three scene-linked symbology conditions and the superimposed symbology HUD condition, $W_{\text {obe }} 1.28<W_{\text {cir }}$ $2.17, q_{\text {.os }}(4,52)$. Finally, a t-test comparing the superimposed symbology and no-gauge baseline conditions showed a significant difference, $t(13)=4.794, p<.001$. Thus, as can be seen in Figure 2, altitude performance was equal in all conditions with altitude gauge symbology, whether scenelinked or superimposed, and better than when only natural, perspective cues were available (the no-gauge baseline condition).

\section{Path performance}

An ANOVA revealed a main effect of altitude gauge format on path performance, $F(4,52)=9.280, p<.001$. Newman-Keuls planned comparisons showed there was no significant difference among the three scene-linked symbology conditions, $\mathrm{W}_{\text {obr }} 2.08<\mathrm{W}_{\text {crit }} 4.74, \mathrm{q}_{.05}(3,52)$. However, there was a significant difference between the superimposed symbology and the three scene-linked symbology conditions, $W_{\text {obe }} 6.69>$ $\mathrm{W}_{\text {crit. }} 5.19, \mathrm{q}_{\mathrm{os}}(4,52)$. Finally, a t-test comparing the superimposed symbology and no-gauge baseline conditions was significant, $t(13)=2.277, p<.04$. Thus, path performance was worst in the superimposed symbology condition, but intermediate in the no-gauge baseline condition, where only natural perspective cues were present. Performance was equal, and better than baseline (no-gauge) in all three scene-linked symbology conditions (see Figure 3).

\section{DISCUSSION}

The purpose of this study was to evaluate altematives to an attentional grouping explanation of the performance benefits that have been found with scene-linked symbology. In a simulated flight environment, concurrent altitude and path maintenance performance was measured with various configurations of altitude gauge formats. Consider first the altitude/path performance tradeoff when comparing the fixedlocation superimposed symbology and the no-gauge baseline conditions: Altitude performance is better with the superimposed symbology but path performance is worse. In contrast, all three scene-linked symbology conditions yield not only better altitude performance compared to the no-gauge baseline condition, but also better path performance. Thus, the present study replicated the super-performance benefit (i.e., better performance than the no-gauge baseline condition) of the scene-linked symbology on path maintenance found by Foyle, McCann and Shelden (1995).

Consider the lack of difference among the three scene-linked symbology conditions for both the path and altitude tasks. As outlined in the introduction, the better-defined path and yisual proximity explanations of the super-performance benefit of 
scene-linked symbology predicted that performance would be best in the on-path scene-linked symbology condition compared to all others. Alternatively, the attentional grouping explanation proposed by Foyle, McCann and Shelden (1995) predicted equal performance among the three scene-linked symbology conditions, and superior path performance with all scene-linked symbology conditions compared to the superimposed symbology and no-gauge conditions. The results of the study clearly support the attentional grouping explanation over the altematives.

The findings of this experiment further our understanding of the effects of scene-linked HUD symbology. Foyle, McCann and Shelden (1995) argued that scene-linked HUD symbology affords more efficient joint processing of the gauges and the world because the symbology attentionally groups with the out-the-window visual scene. The fact that scene-linking the altitude gauges removed the altitude/path performance tradeoff found with a fixed-location, superimposed symbology gauge was consistent with this explanation. It was not clear, however, whether the placement of the scene-linked gauges directly along the path played a role in eliminating the tradeoff. The results of the present experiment answer that it did not: Path and altitude scores were equivalent among the three scenelinked symbology conditions. We conclude that scene-linked altitude gauges support efficient joint processing of the altitude information and the far domain even when the gauges are not located directly along the path.

From an information processing perspective, however, a full understanding of the scene-linked performance benefit has yet to be achieved. One possibility is that scene-linking only encourages a partial division of attention between altitude gauges and the far domain, which yields a more efficient serial extraction of path-related and altitude-related information than in the superimposed condition (i.e., processing is still serial but with reduced switching time). Another possibility is that scene-linking produces a complete division of attention, enabling fully parallel perceptual processing of task-relevant information in the scene-linked symbols and the far domain.

Still another possibility is that scene-linked performance benefits reflect more than just an increase in the efficiency of perceptual processing. Suppose that scene-linking also supports a cognitive integration of the two tasks so that they become, in effect, one task rather than two. More specifically, information regarding the vertical and lateral position of the aircraft might be combined into a single representation, which is then used to null both vertical and lateral error with a single action. Intuitively, such a strategy seems well-suited to produce the level of joint improvement in path and altitude maintenance observed with scene-linked symbology.

This possibility is only speculative, of course, but there is precedent for linking dramatic improvements in multitask performance with post-perceptual levels of task integration. Fagot and Pashler (1992) found dual-task interference of hundreds of milliseconds between two separate responses when the responses were made to two separate features of a single object. However, when two equally distinct responses were made to a single feature of the object, the dual-task interference was virtually eliminated. Fagot and Pashler argued that basing both responses on the same feature enabled them to be selected with a single conjoint operation, thereby reducing two independent response selections to one. Whether the present results with scene-linked symbology reflect a similar phenomenon, or are strictly perceptual in nature, is a matter for future research.

\section{REFERENCES}

Boucek, G. P., Pfaff, T. A., and Smith, W. D. (1983). The use of holographic head-up display of flight path symbology in varying weather conditions (SAE Technical Paper Series 831445). Warrendale, PA: Society of Automotive Engineers, Inc.

Fagot, C., and Pashler, H. (1992). Making two responses to a single object: Implications for the central attentional bottleneck. Journal of Experimental Psychology: Human Perception and Performance, 18, 1058-1079.

Fischer, E., Haines, R. F., and Price, T. A. (1980). Cognitive issues in Head-Up Displays (NASA Technical Paper 1711), NASA: Washington, D. C.

Foyle, D. C., McCann, R. S., and Shelden, S. G. (1995). Attentional issues with superimposed symbology: Formats for scene-linked displays. In R. S. Jensen \& L.A. Rakovan (Eds.), Proceedings of the Eighth International Symposium on Aviation Psychology, 98-103, Columbus, Ohio State University.

Kahneman, D., and Henik, A. (1981). Perceptual organization and attention. In M. Kubovy \& J. Pomerantz (Eds.), Perceptual Organization, 181-211. Hillsdale, NJ: Erlbaum.

Martin-Emerson, R., and Wickens, C. D. (1997). Superimposition, symbology, visual attention, and the head-up display. Human Factors, 39, 581-601.

McCann, R. S., and Foyle, D. C. (1995). Scene-linked symbology to enhance situation awareness. In Situation Awareness: Limitations and Enhancement in the Aviation Environment (AGARD CP \#575), 16:1-16:11.

Pashler, H. (1994). Dual-task interference in simple tasks: Data and theory. Psychological Bulletin, 116, 220-244.

Weinstein, L. F., Ercoline, W. R., Evans, R. H., and Bitton, D. F. (1992). Head-up display standardization and the utility of analog vertical velocity information during instrument flight. The International Journal of Aviation Psychology. 2, 245-260.

Wickens, C. D., and Long, J. (1994). Conformal symbology, attention shifts, and the head-up display. Proceedings of the $38^{4}$ Annual Meeting of the Human Factors and Ergonomics Society, 6-10. Santa Monica, CA: Human Factors and Ergonomics Society. 